\title{
Nested PCR Analysis of Plasmodium Parasites
}

\section{Georges Snounou and Balbir Singh}

\section{Introduction}

In the natural and untreated intermediate hosts, Plasmodium infections are maintained for extended and often lifelong periods. Parasitemia generally reaches its highest level in the first peak after inoculation, and only rises thereafter during increasingly brief and interspersed episodes. As the host acquires tolerance to higher numbers of multiplying parasites, the severity and duration of the clinical episodes that coincide with the parasite peaks diminish.

Microscopic examination of blood has until recently been the sole rapid and practical method to detect and identify Plasmodium parasites unequivocally. The main limitation of this method is the difficulty in detecting very low levels of parasites. In human infections, the probability of detecting the parasite diminishes rapidly as the parasitemia falls below $0.0005 \%$ or 25 parasites/microliter of blood 2.5 parasites per 800 white blood cells counted, assuming $8 \times 10^{3}$ white blood cell and $5 \times 10^{6}$ red blood cell per microliter of blood). Medically, this relative insensitivity is of little consequence because clinical episodes are only rarely associated with such low parasitemias. However, the fact that circulating parasites are undetectable throughout the major part of the infection is of some consequence to the epidemiological and biological perceptions of malaria (1-8).

Amplification of DNA by the polymerase chain reaction (PCR) has provided the opportunity to devise highly sensitive methods of parasite detection (9-16), and the specificity inherent to this method allows the unequivocal identification of the parasite species. The efficiency of the assay is markedly improved when a nested PCR strategy is adopted (13,17-20). In this strategy, two rounds of amplification are carried out, with the product of the first reaction serving as the template for a second reaction where the oligonucleotide primers used hybridize to sequences contained within that product. In this manner, a single parasite genome can be detected routinely and reproducibly, and the sensitivity then depends solely on the quantity and nature of the initial DNA template. A single parasite can be reproducibly detected in the DNA template directly purified from $10 \mu \mathrm{L}$ of blood $(0.000002 \%$ parasitemia $)$. When substantially higher volumes of blood are used, the host's genomic DNA might adversely affect the efficacy of amplification. Removal of the white blood cells before DNA purification would overcome this problem, and thus substantially lower parasitemias would become amenable to PCR analysis. Amplification assays are also suited to the detection and species identification of Plasmodium parasites in the insect vector (21-27). This provides a 